\title{
Next Generation Transit Survey (NGTS)
}

\author{
Peter J. Wheatley ${ }^{1} \dagger$, Don L. Pollacco ${ }^{1}$, Didier Queloz ${ }^{2}$, Heike \\ Rauer $^{3,4}$, Christopher A. Watson ${ }^{5}$, Richard G. West ${ }^{1}$, Bruno \\ Chazelas $^{2}$, Tom M. Louden ${ }^{1}$, Nigel Bannister ${ }^{6}$, Joao Bento ${ }^{1}$, Matthew \\ Burleigh $^{6}$, Juan Cabrera ${ }^{3}$, Philipp Eigmüller ${ }^{3}$, Anders Erikson ${ }^{3}$, \\ Ludovic Genolet ${ }^{2}$, Michael Goad ${ }^{6}$, Andrew Grange ${ }^{6}$, Andrés Jordán ${ }^{7}$, \\ Katherine Lawrie ${ }^{6}$, James McCormac ${ }^{8}$, Marion Neveu ${ }^{2}$ and \\ Simon Walker ${ }^{1}$ \\ ${ }^{1}$ Department of Physics, University of Warwick, Coventry CV4 7AL, UK \\ ${ }^{2}$ Observatoire Astronomique de l'Université de Genève, 1290 Sauverny, Switzerland \\ ${ }^{3}$ Institut für Planetenforschung, Deutsches Zentrum für Luft- und Raumfahrt, Berlin, Germany \\ ${ }^{4}$ Zentrum für Astronomie und Astrophysik, Technische Universität Berlin, Berlin, Germany \\ ${ }^{5}$ Astrophysics Research Centre, Queens University Belfast, Belfast BT7 1NN, UK \\ ${ }^{6}$ Department of Physics and Astronomy, University of Leicester, Leicester LE1 7RH, UK \\ ${ }^{7}$ Departamento de Astronomía y Astrofísica, Pontificia Universidad Católica de Chile, Chile \\ ${ }^{8}$ Isaac Newton Group of Telescopes, 38700 Santa Cruz de la Palma, Canary Islands, Spain
}

\begin{abstract}
The Next Generation Transit Survey (NGTS) is a new ground-based survey for transiting exoplanets. Our primary goal is to find the first statistically-significant sample of Neptunes and super-Earths that are bright enough for radial velocity confirmation. By measuring precise masses and radii we will constrain the bulk composition and internal structure of planets that span the transition between the gas giants and terrestrial planets. Our brightest exoplanets will also be suitable for atmospheric characterisation with large facilities such as the VLT, JWST and the E-ELT. NGTS construction began in June 2013, and the survey is due to commence in 2014 .
\end{abstract}

Keywords. planetary systems, instrumentation: photometers, techniques: photometric, telescopes, surveys

Results from Kepler demonstrate that sub-Neptune sized planets are common (e.g. Borucki et al. 2010), however Kepler has surveyed only one quarter of one percent of the sky, leaving most bright examples of such systems undiscovered. Brighter systems are required for radial-velocity mass determinations that provide precise densities and hence constrain bulk compositions. The brightest systems are also the most prized for follow up measurements of thermal emission, transmission spectra, and spin-orbit alignment.

The Next Generation Transit Survey (NGTS) is a new ground-based sky survey that builds on the legacy of the WASP survey (Pollacco et al. 2006) and aims to find bright systems containing transiting Neptunes and super-Earths. The NGTS facility will consist of an array of twelve $20 \mathrm{~cm} \mathrm{f} / 2.8$ telescopes, each equipped with a large-format redsensitive deep-depletion CCD camera and mounted on its own equatorial fork mount. The instantaneous total field of view of the facility is 96 square degrees, and so our baseline survey of four fields each year for four years will cover 15 times the sky area of Kepler. The design of the facility is described in more detail by Chazelas et al. 2012 .

\section{$\dagger$ Email: P.J.Wheatley@warwick.ac.uk}




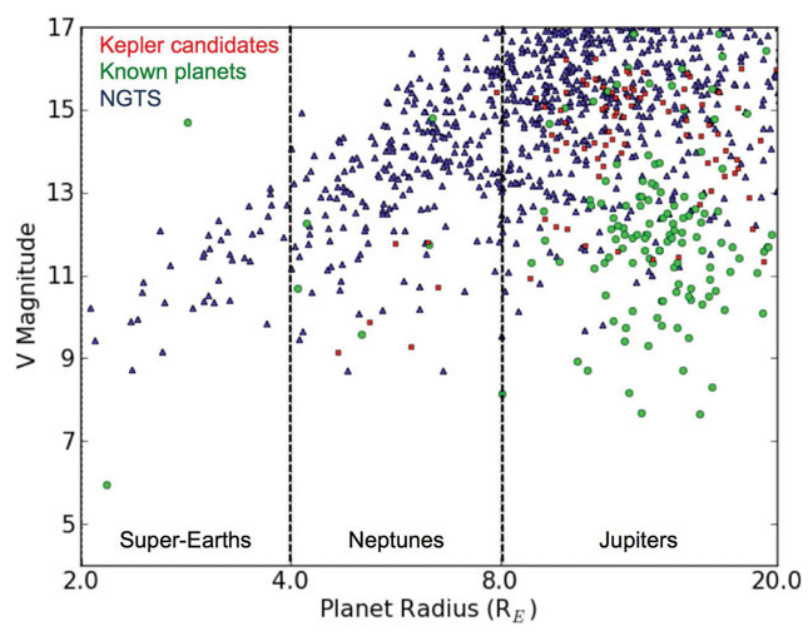

Figure 1. Our simulated population of NGTS planets that can be confirmed in $10 \mathrm{~h}$ with HARPS or ESPRESSO (triangles). These are compared with the known transiting planets with radial-velocity confirmation (circles) and the Kepler candidates that are confirmable with HARPS-N (squares). This simulation shows a total of 39 confirmable super-Earths from NGTS and 231 Neptunes.

Construction of the NGTS facility began in June 2013 at the ESO Paranal observatory, which is the site of the VLT and close to the planned site of the E-ELT. NGTS will benefit from the superb photometric conditions of this site as well as synergy with ESO instrumentation for radial-velocity confirmation and atmospheric characterisation.

The design of the NGTS facility has been validated through the operation of a prototype instrument on La Palma during the winter of 2009/2010. This instrument demonstrated that sub-mmag photometry could be achieved over wide fields from the ground. A complete unit of the NGTS facility has also been assembled and tested in Geneva during 2012 and 2013.

Detailed simulations have been carried out of the sensitivity and likely planet catch of the NGTS survey. Figure 1 shows the simulated catch of NGTS planets that can be confirmed in 10 hours each with either HARPS or ESPRESSO. These simulations are based on our instrument design, prototype performance, the Besançon model of the Galaxy, the weather statistics at the Paranal site and Kepler planet occurrence rates. Over 30 super-Earths and 200 Neptunes from NGTS are predicted to be confirmable (triangles), compared with only a handful from Kepler (squares). These simulations are described in more detail by Wheatley et al. 2013.

The smallest NGTS confirmed planets will be prime targets for the ESA S Mission CHEOPS, which will provide precise radii and hence densities of our super-Earths. As well as testing models of bulk composition of super-Earths, this will allow us to prioritise objects by scale height for atmospheric follow up with VLT and HST, and eventually E-ELT and JWST (as well as possible dedicated missions such as EChO).

\section{References}

Borucki, W. J., Koch, D., Basri, G., et al. 2010, Science, 327, 977

Chazelas, B., Pollacco, D., Queloz, D., et al. 2012, Proceedings of the SPIE, 8444

Pollacco, D. L., Skillen, I., Collier Cameron, A., et al. 2006, PASP 118, 1407

Wheatley, P. J., Pollacco, D. L., Queloz, D., et al. 2013, European Physical Journal Web of Conferences, 47, 13002 